\title{
INDICADORES BIBLIOMÉTRICOS DE LAS PUBLICACIONES CIENTIIFICAS DE LA REVISTA PERUANA DE MEDICINA EXPERIMENTAL Y SALUD PÚBLICA, 2010-2017
}

\author{
Franco Romaní ${ }^{1, a}$, César Cabezas $2, b$
}

\begin{abstract}
RESUMEN
Objetivos. Caracterizar mediante indicadores bibliométricos las publicaciones científicas de la Revista Peruana de Medicina Experimental y Salud Pública (RPMESP) en el periodo 2010-2017. Material y métodos. Estudio bibliométrico, las publicaciones fueron recuperadas de la base de datos Scopus. Los indicadores bibliométricos de producción fueron: número de publicaciones por año y tipo de publicación. Se consideró a los artículos originales y originales breves como unidades de análisis para el número de autores, filiación institucional y país de autor corresponsal, tipo de investigación y diseño de estudio. Los indicadores de impacto fueron: número de citas por publicación según Scopus, métricas de impacto como el CiteScore, Scimago Journal \& Country Rank (SJR) y SciELO Salud Pública. Resultados. Un total de 1045 publicaciones fueron realizadas por la RPMESP. El 40,1\% de las publicaciones correspondieron a artículos originales y originales breves, 1837 autores contribuyeron con dichas publicaciones. 134 filiaciones institucionales fueron declaradas por los autores corresponsales. 48,0\% fueron investigaciones sobre determinantes de un problema de salud, por otro lado, 90,5\% correspondieron a estudios observacionales. Las publicaciones analizadas recibieron 945 citas, de las cuales 78,5\% fueron de publicaciones del periodo 2010-2013. Conclusiones. Cuatro de diez publicaciones de la RPMESP corresponden a artículos originales u originales breves. Las métricas de impacto de la RPMESP según diversas fórmulas de cálculo muestran una tendencia creciente, sin embargo, su magnitud es menor comparada con otras revistas de alcance regional.
\end{abstract}

Palabras clave: Bibliometría; Factor de Impacto; Artículo de Revista; Perú (fuente: DeCS BIREME).

\section{BIBLIOMETRIC INDICATORS OF PAPERS PUBLISHED IN REVISTA PERUANA DE MEDICINA EXPERIMENTAL Y SALUD PÚBLICA, 2010-2017}

\begin{abstract}
Objectives. To characterize, by means of bibliometric indicators, the scientific publications of the Peruvian Experimental Medicine and Public Health Journal, 2010-2017. Materials and Methods. A bibliometric study in which the publications were retrieved from the data base of Scopus. The bibliometric production indicators were: number of publications per year and type of publication. Brief original and original articles were considered as units of analysis based on number of authors, institutional affiliation, and country of corresponding author, type of research and study design. The impact indicators were: number of citations by publication according to Scopus, metric of impact, such as CiteScore, Scimago Journal \& Country Rank (SJR), and SciELO Public Health. Results. A total of 1,045 publications were made by the RPMESP: $40.1 \%$ of publications corresponded to original articles and original briefs; 1,837 authors contributed with these publications; 134 institutional affiliations were declared by the corresponding authors; $48,0 \%$ were research works on determinants of a health problem; on the other hand, $90.5 \%$ corresponded to observational studies. The publications analyzed received 945 citations, of which $78.5 \%$ were for publications for the $2010-2013$ period. Conclusions. Four of ten publications of the RPMESP correspond to brief original or original articles. According to diverse formulas of calculation, the impact metric of the RPMESP shows an ascending trend; however, their magnitude is lower versus other regional journals.
\end{abstract}

Keywords: Bibliometrics; Journal Impact factor; Journal Article; Peru (source: MeSH NLM).

\footnotetext{
Oficina General de Investigación y Transferencia Tecnológica, Instituto Nacional de Salud. Lima, Perú.

Centro Nacional de Salud Pública, Instituto Nacional de Salud. Lima, Perú.

Médico cirujano; ${ }^{\mathrm{b}}$ médico infectólogo.

Recibido: 19/07/2018 Aprobado: 28/11/2018 En línea: 21/12/2018
}

Citar como: Romaní F, Cabezas C. Indicadores bibliométricos de las publicaciones científicas de la Revista Peruana de Medicina Experimental y Salud Pública, 2010-2017. Rev Peru Med Exp Salud Publica. 2018;35(4):620-9. doi:10.17843/rpmesp.2018.354.3817. 


\section{INTRODUCCIÓN}

La Revista Peruana de Medicina Experimental y Salud Pública (RPMESP) es el órgano oficial de difusión científica del Instituto Nacional de Salud (INS) del Perú. Inició su publicación en octubre de 1942 hasta 1960 con el nombre de Revista de Medicina Experimental, luego de un periodo de inactividad vuelve a editarse en 1997 y hasta la fecha es publicada de manera ininterrumpida. El 2001, adopta su denominación actual y a partir del 2002 tiene periodicidad trimestral. Desde 2010, la revista se encuentra indizada en las bases de datos Scopus y Medline, también se encuentra indizada en SciELO Salud Pública, y es de acceso libre, permanente y no tiene cargos por publicación.

La bibliometría tiene entre sus aplicaciones la evaluación de la producción científica de un país, de una institución o de una revista científica (1); por ello, diversas revistas han analizado sus publicaciones mediante indicadores bibliométricos con la finalidad de observar sus tendencias y características ${ }^{(2-4)}$, o medir la influencia o impacto de sus publicaciones ${ }^{(5)}$; para de esta manera generar evidencias en pro de mejorar su gestión editorial. Por otro lado, la bibliometría de una revista permite a los investigadores conocer los indicadores bibliométricos y características de las publicaciones de las revistas, información que es valiosa y relevante para que los autores decidan el envío de algún manuscrito.

El último análisis de la producción, visibilidad y citación de la RPMESP correspondió al periodo 2002-2009 (6); sin embargo, el 2010 la indización en las bases de datos Scopus y Medline ha permitido incrementar la visibilidad de la RPMESP. Por ello, es pertinente describir la producción científica de la revista biomédica peruana de mayor visibilidad en los últimos años, dicho conocimiento puede generar evidencias para implementar ajustes en las políticas editoriales de la RPMESP, y potencialmente en otras revistas científicas biomédicas peruanas.

El objetivo del presente estudio es cuantificar y caracterizar los indicadores bibliométricos de producción y citación de las publicaciones científicas de la RPMESP en el periodo 2010 a 2017.

\section{MATERIAL Y MÉTODOS}

\section{DISEÑO Y UNIDADES DE ANÁLISIS}

Se realizó un estudio descriptivo mediante un análisis bibliométrico de las publicaciones de la RPMESP para el periodo 2010-2017. Las publicaciones fueron recuperadas de la base de datos Scopus. Dicha base fue seleccionada debido a que el $85 \%$ de sus publicaciones están indizadas con vocabulario controlado; además, permite exportar información sobre métricas, incluyendo número de citas. La RPMESP se encuentra indizada en Scopus desde el 2010, asegurando la recuperación de todas las publicaciones para el periodo de estudio.

\section{MENSAJES CLAVE}

Motivación para realizar el estudio. La Revista Peruana de Medicina Experimental y Salud Pública (RPMESP) es la revista biomédica peruana de mayor difusión, desde el 2010 se encuentra indizada en Scopus y Medline. No se cuenta con los principales indicadores bibliométricos de la revista, considerada como referente nacional, en los últimos ocho años. Esta información es relevante para la gestión editorial de la revista y para los autores interesados en publicar en la RPMESP.

Principales hallazgos.La RPMESP publicó 1045 documentos entre 2010 y 2017, de los cuales $40,1 \%$ fueron artículos originales u originales breves. Un total de 1837 autores contribuyeron en las publicaciones originales, las cuales recibieron 945 citas.

Implicancias. La RPMESP ha incrementado el número de publicaciones científicas. Constituye un medio de difusión científica para principalmente autores nacionales. La mayor parte de publicaciones fueron estudios observacionales y de carácter epidemiológico. Las métricas de impacto de la revista muestran un patrón ascendente.

Un investigador con experiencia en estudios bibliométricos (FRRR) realizó la búsqueda en Scopus en mayo del 2018. Se utilizó la búsqueda avanzada con los términos de búsqueda «SRCTITLE (Revista peruana de medicina experimental y salud pública)» y se usó el filtro temporal para el periodo 2010 al 2017. Se exportaron los resultados en formato csv, luego fue importado en una base de datos en Microsoft Excel@ para limpieza y normalización, las cuales fueron realizadas manualmente por el primer autor. Para la exportación, fueron seleccionados las siguientes variables: autor(es), título de documento, año, volumen, número y página, número de citas, tipo de documento, filiaciones y dirección de correspondencia.

Las unidades de análisis para la caracterización bibliométrica fueron las publicaciones originales es decir «artículo original» y «original breve», pues corresponden a estudios de investigaciones primarias o secundarias. Las revisiones, simposio, sección especial, reporte de casos, historia de la salud pública, galería fotográfica y carta al editor fueron excluidas.

\section{VARIABLES DE ESTUDIO}

El tipo de publicación fue según las secciones de la RPMESP (artículo original, original breve, revisión, simposio, sección especial, reporte de casos, historia de la salud pública, galería fotográfica y carta al editor). La verificación del tipo de publicación fue realizada ingresando al texto completo en la página web de la RPMESP (http://www.rpmesp.ins. gob.pe/index.php/rpmesp/issue/archive).

Para las publicaciones originales se obtuvo el número de autores por publicación, filiación institucional y filiación país del autor corresponsal. El tipo de investigación (estudios 
para medir la magnitud y distribución de problemas de salud, estudio de determinantes de problemas de salud, estudios de desarrollo de soluciones o tecnologías en salud, estudios de traslado de soluciones o evidencia en políticas y servicios de salud, y evaluaciones de impacto de intervenciones) ${ }^{(7)}$ y diseño de estudio (observaciones, experimentales, revisiones sistemáticas/metaanálisis) fueron asignadas por un investigador con experiencia en estudios bibliométricos (FRRR), luego de la revisión de la sección material y métodos de la publicación en texto completo. El índice de productividad fue calculado como el logaritmo natural del número de publicaciones originales en un año.

Las métricas de impacto corresponden a la RPMESP, es decir consideran todos sus tipos de publicaciones. Se utilizó el CiteScore de Scopus, el cual se calcula a partir del número de citas recibidas por una revista científica en un año por documentos publicados en los tres años previos, dividido por el número de documentos publicados e indizados en Scopus en los mismos tres años. El CiteScore utiliza todos los tipos de documentos incluidos en Scopus (https://goo.gl/8ZGb4Y).

También se recuperó la métrica del Scimago Journal \& Country Rank (SJR), la cual es estimada a partir de la base de datos de Scopus, pero otorgando un peso determinado a las citas de una revista, en función de su área científica y su relevancia (medida a partir de su SJR). El cálculo usa una ventana de citación de tres años y las auto citas están restringidas (https://goo.gl/1CrHL6). El indicador de impacto de SciELO puede ser calculado para dos y tres años, se basa en el número de citas recibidas y número de artículos publicados única y exclusivamente en revistas registradas en esta base de datos. Se presentará este indicador calculado para tres años, para fines de comparación.
El control de calidad de los datos fue realizado por el autor principal, consistió en eliminar publicaciones repetidas, verificar la adecuada clasificación de publicaciones según tipo de publicación por sección de la RPMESP, el diseño de estudio y tipo de investigación.

\section{ANÁLISIS DE DATOS}

Realizamos el análisis descriptivo usando frecuencias absolutas y relativas de los tipos de publicación por año de estudio. Para las publicaciones originales se analizó la frecuencia de filiaciones institucionales y país del autor corresponsal, del tipo de publicación y diseño del estudio. Las variables cuantitativas como número de autores y número de citas fueron analizadas mediante la media y desviación estándar. El análisis de producción científica fue para todo el periodo, y para los subperiodos 20102013 y 2014-2017. El análisis estadístico fue realizado en

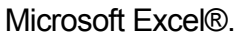

\section{RESULTADOS}

\section{CARACTERÍSTICAS GENERALES}

En el periodo 2010-2017, la RPMESP publicó cuatro números anuales. Luego del control de calidad de los datos, seis registros (exportados por un error informático a partir de Scopus) fueron eliminados por no corresponder a publicaciones incluidas en la RPMESP. Un total de 1045 publicaciones fueron incluidas en el análisis inicial, de las cuales $26,1 \%$ fueron artículos originales, $21,7 \%$ fueron cartas al editor y $14,0 \%$ fueron originales breves, estas concentran el $61,8 \%$ de las publicaciones (Tabla 1 ).

Tabla 1. Tipo de publicaciones en la Revista Peruana de Medicina Experimental y Salud Pública, periodo $2010-2017$.

\begin{tabular}{|c|c|c|c|c|c|c|c|c|c|c|}
\hline \multirow{2}{*}{ Tipo de publicación } & \multicolumn{8}{|c|}{ Año } & \multirow{2}{*}{ Total } & \multirow{2}{*}{$\%$} \\
\hline & 2010 & 2011 & 2012 & 2013 & 2014 & 2015 & 2016 & 2017 & & \\
\hline Artículo original & 25 & 35 & 27 & 25 & 44 & 42 & 43 & 32 & 273 & 26,1 \\
\hline Carta al editor & 23 & 25 & 26 & 42 & 30 & 36 & 24 & 21 & 227 & 21,7 \\
\hline Original breve & 12 & 17 & 11 & 25 & 18 & 24 & 17 & 22 & 146 & 14,0 \\
\hline Simposio & 21 & 14 & 15 & 17 & 14 & 13 & 18 & 12 & 124 & 11,9 \\
\hline Sección especial & 7 & 9 & 13 & 12 & 12 & 9 & 10 & 11 & 83 & 7,9 \\
\hline Editorial & 6 & 7 & 8 & 7 & 5 & 6 & 7 & 6 & 52 & 5,0 \\
\hline Reporte de caso & 4 & 4 & 6 & 7 & 9 & 8 & 5 & 7 & 50 & 4,8 \\
\hline Artículo de revisión & 7 & 5 & 3 & 9 & 7 & 7 & 5 & 4 & 47 & 4,5 \\
\hline Galería fotográfica & 4 & 4 & 4 & 2 & 2 & 0 & 3 & 1 & 20 & 1,9 \\
\hline Historia de la Salud Pública & 0 & 0 & 2 & 4 & 4 & 0 & 1 & 3 & 14 & 1,3 \\
\hline Personalidades destacadas & 4 & 4 & 0 & 0 & 0 & 0 & 0 & 0 & 8 & 0,8 \\
\hline Erratum & 0 & 0 & 0 & 0 & 0 & 0 & 1 & 0 & 1 & 0,1 \\
\hline Total & 113 & 124 & 115 & 150 & 145 & 145 & 134 & 119 & 1045 & 100,0 \\
\hline
\end{tabular}


La proporción de investigaciones originales (artículo original u original breve) sobre el total de publicaciones fue de $32,7 \%$ (2010), 41,9\% (2011), 33,0\% (2012), 33,3\% (2013), 42,8\% (2014), 45,5\% (2015), 44,8\% (2016) y 45,4\% (2017). Para todo el periodo de estudio dicha proporción fue $40,1 \%$, para el sub periodo 2010-2013 la proporción fue de 35,3\%, mientras que en el periodo 2014-2017, la proporción fue de 44,6\% (Tabla 1).

\section{INDICADORES DE PRODUCCIÓN DE INVESTIGACIONES ORIGINALES}

El promedio del índice de productividad de investigaciones originales por año fue de 3,94, con un mínimo de 3,61 para el 2010, y máximo de 4,19 para 2015. El promedio del índice de productividad para el sub periodo 2010-2013 fue 3,78 , para el sub periodo $2014-2017$ fue 4,10 .

Para el periodo estudiado, 1837 autores contribuyeron con artículos originales $(n=1209)$ u originales breves $(n=628)$. La media de autores por artículo fue de $5,02 \pm 2,81$, la mediana fue de 4,5, el mínimo de 1 y máximo de 26 autores. Para los artículos originales la media de autores fue de $5,04 \pm 2,97$, para originales breves fue de 4,98 $\pm 2,47$ autores.

Un total de 134 filiaciones institucionales diferentes fueron declaradas por los autores corresponsales de investigaciones originales (Tabla 2). En el sub periodo 2010-2013, dicho número fue de 68, y para el sub periodo 2014-2017 el número de filiaciones institucionales del autor corresponsal incrementó a 87.

Según la filiación país del autor corresponsal, 18 países contribuyeron con publicaciones originales. El país más representado fue Perú con 334 (79,7\%), seguidos por
Colombia ( $n=17 ; 4,1 \%)$, Argentina ( $n=12 ; 2,9 \%)$, Venezuela $(n=10 ; 2,4 \%)$, España $(n=7 ; 1,7 \%)$, Chile $(n=7 ; 1,7 \%)$, México $(n=6 ; 1,4 \%)$, Estados Unidos $(n=6 ; 1,4 \%)$, Cuba $(n=5$; $1,2 \%)$, Ecuador $(n=4 ; 1,0 \%)$, Brasil $(n=3 ; 0,7 \%)$, Guatemala $(n=2 ; 0,5 \%)$, y Australia, Bolivia, Canadá, Israel, Paraguay y Portugal con una publicación $(0,2 \%)$.

Asimismo, 201 (48,0\%) publicaciones originales fueron investigaciones sobre los determinantes de un problema de salud, y el 37,9\% fueron investigaciones para medir la magnitud y distribución de un daño a la salud. Respecto al diseño, los estudios observacionales fueron los más frecuentemente publicados $(90,5 \%)$ (Tabla 3$)$.

\section{ANÁLISIS DE CITACIONES DE PUBLICACIONES ORIGINALES}

El total de citaciones en el periodo analizado es de 945 , de las cuales $742(78,5 \%)$ corresponden a publicaciones entre 2010 y 2013. Entre 2010 y 2017, 167 (39,9\%) publicaciones originales no cuentan con citas, de ellas 90 fueron publicaciones del 2016 y 2017. En el subperiodo 2010-2013, $25(14,1 \%)$ publicaciones no cuentan con citas, mientras que para 2014-2017, $142(58,7 \%)$ no cuentas con citas (Tabla 4).

Un total de $78(18,6 \%)$ publicaciones fueron citadas una vez, $57(13,6 \%)$ dos veces, y sólo una publicación recibió $22,23,25$ y 35 citas. Además, 18 publicaciones originales recibieron diez o más citaciones, todas fueron publicadas en el subperiodo 2010-2013. Seis publicaciones tuvieron como filiación institucional corresponsal al INS, y en cinco la filiación correspondió a la Universidad Peruana Cayetano Heredia (UPCH) (Tabla 5).

Tabla 2. Filiación institucional de autores corresponsales con cuatro o más publicaciones originales en la Revista Peruana de Medicina Experimental y Salud Pública en el periodo 2010-2017.

\begin{tabular}{|c|c|c|c|c|c|c|c|c|c|}
\hline \multirow{2}{*}{ Filiación Institucional Corresponsal } & \multicolumn{8}{|c|}{ Año } & \multirow{2}{*}{ Total } \\
\hline & 2010 & 2011 & 2012 & 2013 & 2014 & 2015 & 2016 & 2017 & \\
\hline Universidad Peruana Cayetano Heredia & 6 & 14 & 8 & 8 & 16 & 15 & 11 & 8 & 86 \\
\hline Universidad Nacional Mayor de San Marcos & 9 & 9 & 8 & 6 & 7 & 10 & 5 & 4 & 58 \\
\hline Instituto Nacional de Salud & 7 & 3 & 10 & 6 & 10 & 3 & 3 & 9 & 51 \\
\hline Universidad Peruana de Ciencias Aplicadas & 1 & 1 & 1 & 3 & 4 & 6 & 5 & 2 & 23 \\
\hline Ministerio de Salud del Perú & 1 & 0 & 0 & 2 & 3 & 3 & 2 & 1 & 12 \\
\hline Universidad de Carabobo & 0 & 1 & 0 & 0 & 1 & 2 & 2 & 0 & 6 \\
\hline Universidad de San Martín de Porres & 0 & 0 & 1 & 0 & 0 & 1 & 2 & 2 & 6 \\
\hline Universidad de Buenos Aires & 0 & 0 & 0 & 0 & 0 & 0 & 5 & 1 & 6 \\
\hline Hospital Nacional Cayetano Heredia & 0 & 0 & 0 & 2 & 1 & 1 & 1 & 0 & 5 \\
\hline Universidad Nacional de San Antonio Abad del Cusco & 0 & 2 & 0 & 2 & 1 & 0 & 0 & 0 & 5 \\
\hline Instituto Nacional de Enfermedades Neoplásicas & 0 & 0 & 0 & 0 & 1 & 3 & 0 & 1 & 5 \\
\hline Universidad Tecnológica de Pereira & 0 & 0 & 0 & 3 & 0 & 0 & 1 & 0 & 4 \\
\hline Hospital Nacional Edgardo Rebagliati Martins & 0 & 1 & 1 & 0 & 0 & 0 & 0 & 2 & 4 \\
\hline
\end{tabular}


Tabla 3. Tipo de investigación y diseño de estudio de publicaciones originales de la Revista Peruana de Medicina Experimental y Salud Pública, periodo 2010-2017.

\begin{tabular}{|c|c|c|c|c|c|c|c|c|c|c|}
\hline \multirow{2}{*}{ Variable } & \multicolumn{8}{|c|}{ Año } & \multirow[b]{2}{*}{ Total } & \multirow[b]{2}{*}{$\%$} \\
\hline & 2010 & 2011 & 2012 & 2013 & 2014 & 2015 & 2016 & 2017 & & \\
\hline \multicolumn{11}{|l|}{ Tipo de investigación } \\
\hline $\begin{array}{l}\text { Estudio de magnitud y distribución del } \\
\text { problema de salud }\end{array}$ & 9 & 13 & 2 & 11 & 17 & 32 & 45 & 30 & 159 & 37,9 \\
\hline $\begin{array}{l}\text { Estudio de determinantes de } \\
\text { problema de salud }\end{array}$ & 19 & 32 & 32 & 35 & 34 & 27 & 9 & 13 & 201 & 48,0 \\
\hline $\begin{array}{l}\text { Estudio de desarrollo de soluciones, } \\
\text { intervenciones o tecnologías en salud }\end{array}$ & 8 & 3 & 3 & 2 & 6 & 7 & 5 & 8 & 42 & 10,0 \\
\hline $\begin{array}{l}\text { Estudios para traslado de soluciones } \\
\text { o evidencias en políticas y servicios } \\
\text { de salud }\end{array}$ & 1 & 3 & 0 & 0 & 2 & 0 & 0 & 0 & 6 & 1,4 \\
\hline $\begin{array}{l}\text { Estudio de evaluación de impacto de } \\
\text { intervenciones o soluciones }\end{array}$ & 0 & 1 & 1 & 2 & 3 & 0 & 1 & 3 & 11 & 2,6 \\
\hline \multicolumn{11}{|l|}{ Diseño de estudio } \\
\hline Observacionales & 35 & 49 & 34 & 47 & 54 & 58 & 55 & 47 & 379 & 90,5 \\
\hline Experimentales & 2 & 3 & 3 & 3 & 8 & 8 & 5 & 6 & 38 & 9,1 \\
\hline Revisiones sistemáticas / Meta-análisis & 0 & 0 & 1 & 0 & 0 & 0 & 0 & 1 & 2 & 0,5 \\
\hline
\end{tabular}

\section{MÉTRICAS DE IMPACTO DE LA RPMESP}

El CiteScore de la RPMESP mostró una tendencia creciente entre el 2011 y el 2013, alcanzando un puntaje de 0,49 el 2013; posteriormente entre el 2014 y 2017, de 0,40 incrementó a 0,47. Las magnitudes de las métricas del SJR son menores a las de CiteScore para todos los años, y estos a su vez son menores al indicador de impacto de SciELO (calculado para tres años), a excepción del 2012 (Figura 1).

\section{DISCUSIÓN}

En el periodo 2010-2017, la RPMESP publicó 1045 documentos, lo cual con respecto al periodo 2002-2009 constituye un marcado incremento. El número promedio de publicaciones por año pasó de 62 a 131 entre ambos periodos. En nuestro estudio, cuatro de diez publicaciones correspondieron a artículos originales u originales breves, tipo de publicación que constituye el medio establecido para la comunicación de resultados de investigación; sin embargo, dicha proporción es menor comparada al 55,3\% conseguido por la RPMESP en el octenio 2002-2009 ${ }^{(6)}$. Esta diferencia podría explicarse por el incremento en términos absolutos de otros tipos de publicaciones como las cartas al editor, que fue el segundo tipo más frecuente de publicación entre 2010 y 2017 . El incremento en el número de las cartas al editor se observa desde el 2009 , año en que llegó al $15,2 \%$ del total de publicaciones.

Según el ranking de revistas desarrollado por Scimago, el incremento en el número publicaciones originales es una tendencia en gran parte de las revistas científicas latinoamericanas del ámbito de la salud pública, por ejemplo, en la Revista Panamericana de Salud Pública (RPSP) la proporción de publicaciones originales en el periodo $1997-2002$ fue de $26,9 \%$, y alcanzó el $70,3 \%$ para 2008-2012 ${ }^{(2)}$.

En las 419 publicaciones originales, la media de autores por publicación fue cinco, no encontramos diferencias entre la media de autores para artículos originales $u$ originales breves. Este hallazgo llama la atención debido a que, según las instrucciones para autores de la RPMESP, los originales breves en virtud de sus objetivos, diseño y resultados pueden ser publicados en un formato abreviado de 2000 palabras, características que estarían asociadas a una menor complejidad de la investigación y, por ende, a un menor número de autores ${ }^{(8)}$. Por otro lado, el promedio de autores por publicación en la RPMESP difiere de lo encontrado en otros análisis; entre 1997 y 2012, las publicaciones originales de la RPSP tuvieron una media de autores de 3,86 (2); y entre 1999 y 2006, la revista Journal of Advanced Nursing tuvo 2,45 autores por artículo (9). Estos hallazgos sugieren que existirían dificultades para la adecuada atribución de la autoría entre los autores que publican en la RPMESP, a pesar que las definiciones de autoría se encuentran claramente establecidas por el Comité Internacional de Editores de Revistas Médicas (ICMJE).

Las filiaciones institucionales corresponsales de mayor participación fueron dos universidades (UPCH y Universidad 
Tabla 4. Número de citas de publicaciones originales de la Revista Peruana de Medicina Experimental y Salud Pública en el periodo 2010-2017, según Scopus.

\begin{tabular}{|c|c|c|c|c|c|c|c|c|c|c|}
\hline \multirow{2}{*}{$\begin{array}{l}\text { Número de } \\
\text { citas* }^{*}\end{array}$} & \multicolumn{8}{|c|}{ Año } & \multirow{2}{*}{$\begin{array}{l}\text { Número de } \\
\text { publicaciones }\end{array}$} & \multirow{2}{*}{$\%$} \\
\hline & 2010 & 2011 & 2012 & 2013 & 2014 & 2015 & 2016 & 2017 & & \\
\hline 0 & 2 & 4 & 8 & 11 & 19 & 33 & 45 & 45 & 167 & 39,9 \\
\hline 1 & 2 & 5 & 9 & 7 & 19 & 16 & 12 & 8 & 78 & 18,6 \\
\hline 2 & 10 & 11 & 3 & 10 & 9 & 10 & 3 & 1 & 57 & 13,6 \\
\hline 3 & 2 & 10 & 2 & 12 & 5 & 3 & 0 & 0 & 34 & 8,1 \\
\hline 4 & 2 & 3 & 2 & 3 & 2 & 4 & 0 & 0 & 16 & 3,8 \\
\hline 5 & 1 & 4 & 2 & 4 & 1 & 0 & 0 & 0 & 12 & 2,9 \\
\hline 6 & 3 & 2 & 5 & 2 & 4 & 0 & 0 & 0 & 16 & 3,8 \\
\hline 7 & 3 & 3 & 2 & 1 & 1 & 0 & 0 & 0 & 10 & 2,4 \\
\hline 8 & 2 & 1 & 3 & 0 & 0 & 0 & 0 & 0 & 6 & 1,4 \\
\hline 9 & 1 & 2 & 0 & 0 & 2 & 0 & 0 & 0 & 5 & 1,2 \\
\hline 10 & 3 & 1 & 0 & 0 & 0 & 0 & 0 & 0 & 4 & 1,0 \\
\hline 11 & 1 & 2 & 0 & 0 & 0 & 0 & 0 & 0 & 3 & 0,7 \\
\hline 12 & 2 & 1 & 0 & 0 & 0 & 0 & 0 & 0 & 3 & 0,7 \\
\hline 13 & 0 & 1 & 1 & 0 & 0 & 0 & 0 & 0 & 2 & 0,5 \\
\hline 14 & 2 & 0 & 0 & 0 & 0 & 0 & 0 & 0 & 2 & 0,5 \\
\hline 22 & 0 & 1 & 0 & 0 & 0 & 0 & 0 & 0 & 1 & 0,2 \\
\hline 23 & 0 & 1 & 0 & 0 & 0 & 0 & 0 & 0 & 1 & 0,2 \\
\hline 25 & 1 & 0 & 0 & 0 & 0 & 0 & 0 & 0 & 1 & 0,2 \\
\hline 35 & 0 & 0 & 1 & 0 & 0 & 0 & 0 & 0 & 1 & 0,2 \\
\hline
\end{tabular}

* Consulta en base de datos Scopus: mayo de 2018

Nacional Mayor de San Marcos - UNMSM), ambas también estuvieron entre las cuatro instituciones nacionales de mayor producción científica en el periodo 2000 - $2009{ }^{(10)}$, en dicho análisis las otras dos instituciones con mayor producción fueron el Centro Número seis de Investigación Médico Naval (NAMRU-6) y la Asociación Benéfica Prisma (Prisma); este patrón es coherente con un estudio bibliométrico de la producción científica peruana entre 2007-2011, en el cual las dos universidades con mayor producción fueron UPCH y UNMSM, mientras Prisma y NAMRU-6 lideraron entre instituciones biomédicas ${ }^{(11)}$. En nuestro estudio estas instituciones no se encuentran como las más productivas debido a que sólo analizamos publicaciones en la RPMESP, lo cual no constituye la producción científica biomédica nacional; otra explicación es que hayan participado, pero no como filiación del autor corresponsal.

EI INS, institución que publica la RPMESP, es la tercera institución corresponsal con mayor participación. Este hallazgo contrasta con la RPSP, publicada por la Organización Panamericana de Salud (OPS), y en donde el $24 \%$ de las publicaciones tienen participación de la OPS ${ }^{(2)}$. A pesar que la RPMESP, es el órgano oficial de difusión científica del INS, la mayor cantidad de contribuciones originales provienen de universidades públicas o privadas. El $80 \%$ de las publicaciones tuvieron como filiación país corresponsal al Perú, ello sugiere que la RPMESP es una atractiva alternativa para autores nacionales, debido a su indización en bases como Scopus y Medline, al uso del idioma español y a sus crecientes métricas de impacto. Colombia y Argentina son las filiación país de autor corresponsal con mayor contribución luego de Perú, la participación de otros países a nivel de América del Sur y otras partes del mundo es más dispersa y no llegan al dos por ciento de las publicaciones originales; la menor participación internacional puede ser explicada por la existencia de revistas del campo de salud pública con características que aseguran una mayor visibilidad y citabilidad de las publicaciones, como son el mayor factor de impacto, una mayor visibilidad en bases de datos (incluyendo indización en Web of Science) y que brindan la posibilidad de publicar en varios idiomas, como el inglés o portugués ${ }^{(4,12)}$.

En el periodo analizado, las publicaciones originales provienen en un $85 \%$ de investigaciones epidemiológicas, que incluyen estudios para estimar la magnitud y distribución de problemas de salud, o indagar sobre factores o determinantes de problemas de salud. A pesar que la RPMESP tiene como ámbito la investigación biomédica, en el cual se pueden difundir resultados de investigación 
Tabla 5. Publicaciones originales de la Revista Peruana de Medicina Experimental y Salud Pública con diez o más citaciones en el periodo 2010-2017, según Scopus.

\begin{tabular}{|c|c|c|c|c|}
\hline Primer autor & $\begin{array}{l}\text { Filiación } \\
\text { institucional } \\
\text { (País) }\end{array}$ & Título & Año & $\begin{array}{l}\text { Número } \\
\text { de citas* }\end{array}$ \\
\hline Álvarez-Dongo D. et al. & INS (Perú) & $\begin{array}{l}\text { Sobrepeso y obesidad: Prevalencia y determinantes sociales del } \\
\text { exceso de peso en la población peruana }(2009-2010)\end{array}$ & 2012 & 35 \\
\hline Huamaní C. et al. & UNMSM (Perú) & $\begin{array}{l}\text { Producción científica peruana en medicina y redes de colaboración, } \\
\text { análisis del science citation index 2000-2009 }\end{array}$ & 2010 & 25 \\
\hline Ochoa TJ. et al. & UPCH (Perú) & $\begin{array}{l}\text { Frecuencia y patotipos de Escherichia coli diarrogénica en niños } \\
\text { peruanos con y sin diarrea }\end{array}$ & 2011 & 23 \\
\hline Zevallos L. et al. & UCSUR (Perú) & $\begin{array}{l}\text { Oferta y demanda de médicos especialistas en los establecimientos } \\
\text { de Salud del Ministerio de Salud: Brechas a nivel nacional, por } \\
\text { regiones y tipo de especialidad }\end{array}$ & 2011 & 22 \\
\hline Culqui DR. et al. & INS (Perú) & Tuberculosis en la población indígena del Perú 2008 & 2010 & 14 \\
\hline Moreno N. et al. & $\begin{array}{l}\text { Instituto } \\
\text { Colombiano de } \\
\text { Medicina Tropical } \\
\text { (Colombia) }\end{array}$ & $\begin{array}{l}\text { Aplicación de las pruebas de PCR convencional simple y múltiple } \\
\text { para la identificación de aislamientos de Leptospira spp. en } \\
\text { Colombia }\end{array}$ & 2010 & 14 \\
\hline Lam-Figueroa N. et al. & UNMSM (Perú) & $\begin{array}{l}\text { Adicción a Internet: Desarrollo y Validación de un Instrumento en } \\
\text { escolares adolescentes de Lima, Perú }\end{array}$ & 2011 & 13 \\
\hline Asencios L. et al. & INS (Perú) & $\begin{array}{l}\text { Prueba molecular Genotype } ® M T B D R p l u s, \text { una alternativa para la } \\
\text { detección rápida de tuberculosis multidrogorresistente }\end{array}$ & 2012 & 13 \\
\hline $\begin{array}{l}\text { Choquehuanca-Vilca } \\
\text { V. et al. }\end{array}$ & $\begin{array}{l}\text { Ministerio de } \\
\text { Salud (Perú) }\end{array}$ & $\begin{array}{l}\text { Perfil epidemiológico de los accidentes de tránsito en el Perú, } \\
2005-2009\end{array}$ & 2010 & 12 \\
\hline Miranda M. et al. & INS (Perú) & $\begin{array}{l}\text { Situación de la calidad de agua para consumo en hogares de } \\
\text { niños menores de cinco años en Perú, 2007-2010 }\end{array}$ & 2010 & 12 \\
\hline Mamani E. et al. & INS (Perú) & $\begin{array}{l}\text { Circulación de un linaje diferente del virus dengue } 2 \text { genotipo } \\
\text { América / Asia en la región Amazónica de Perú, 2010] }\end{array}$ & 2011 & 12 \\
\hline Gorriti A. et al. & UNMSM (Perú) & $\begin{array}{l}\text { Toxicidad oral a } 60 \text { días del aceite de sacha inchi (Plukenetia } \\
\text { volubilis } I \text {.) y linaza (Linum usitatissimum I.) y determinación de la } \\
\text { dosis letal } 50 \text { en roedores }\end{array}$ & 2010 & 11 \\
\hline Mayta-Tristán P. et al. & UPC (Perú) & $\begin{array}{l}\text { Proyección de trabajo en el interior del país y factores asociados } \\
\text { en médicos recién colegiados de Lima, Perú } 2010\end{array}$ & 2011 & 11 \\
\hline $\begin{array}{l}\text { Castañeda-Guarderas } \\
\text { A. et al. }\end{array}$ & UPCH (Perú) & $\begin{array}{l}\text { Registro de pacientes con accidente cerebro vascular en un } \\
\text { hospital público del Perú, } 2000-2009\end{array}$ & 2011 & 11 \\
\hline Málaga G. et al. & UPCH (Perú) & $\begin{array}{l}\text { Elevada frecuencia de dislipidemia y glucemia basal alterada en } \\
\text { una población peruana de altura }\end{array}$ & 2010 & 10 \\
\hline Mamani E. et al. & INS (Perú) & $\begin{array}{l}\text { Infecciones concurrentes por dos serotipos del virus dengue } \\
\text { durante un brote en el noroeste de Perú, } 2008\end{array}$ & 2010 & 10 \\
\hline Rosas-Aguirre A. et al. & UPCH (Perú) & $\begin{array}{l}\text { Uso de paneles de láminas estandarizadas para la evaluación } \\
\text { de competencias en el diagnóstico microscópico de malaria en la } \\
\text { Amazonía Peruana }\end{array}$ & 2010 & 10 \\
\hline Rosas-Aguirre A. et al. & UPCH (Perú) & $\begin{array}{l}\text { Posesión, retención y uso de mosquiteros tratados con insecticidas } \\
\text { de larga duración luego de un año de su distribución en Loreto, } \\
\text { Perú. }\end{array}$ & 2011 & 10 \\
\hline
\end{tabular}

INS: Instituto Nacional de Salud; UNMSM: Universidad Nacional Mayor de San Marcos; UPCH, Universidad Peruana Cayetano Heredia; UCSUR: Universidad Científica del Sur; UPC: Universidad Peruana de Ciencias Aplicadas

* Consulta en base de datos Scopus: mayo de 2018.

experimental, sólo una de cada diez publicaciones originales tiene diseño experimental. Dicho hallazgo, es congruente con lo encontrado en estudios vinculados con prioridades de investigación en salud con participación de investigadores peruanos, en dichas publicaciones sólo el 7,4\% fueron estudios experimentales ${ }^{(13)}$.
La mayor proporción de publicaciones citadas pertenece al subperiodo 2010-2013, ello explica por qué las métricas de impacto de la RPMESP alcanzaron su punto más alto el 2013. Es destacable que entre el 2010 y 2012 cuatro manuscritos recibieron más de 20 citaciones cada uno; ello podría reflejar un adecuado trabajo en la selección 


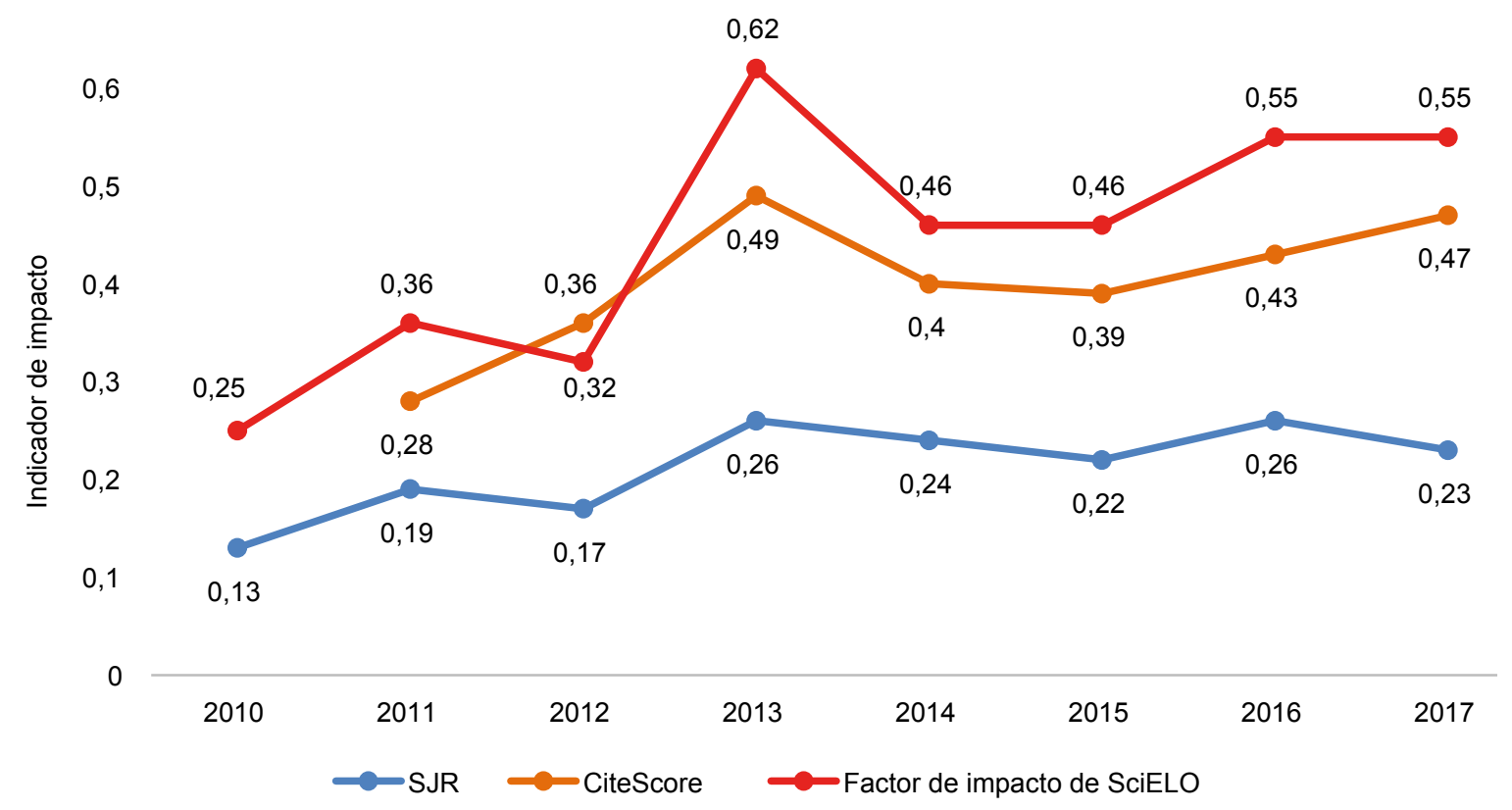

Figura 1. Indicadores de impacto de la Revista Peruana de Medicina Experimental y Salud Pública según diversas fuentes para el periodo 2010-2017.

Fuente: Scimago Journal \& Country Rank (SJR), CiteScore, SciELO

y publicación de dichos manuscritos ${ }^{(5)}$, así como, el efecto del transcurso de los años a partir de su publicación lo cual incrementa la posibilidad de citación ${ }^{(14)}$. Entre las 18 publicaciones más citadas destacan cuatro estudios que estandarizan y validan alguna tecnología de uso en salud pública, este tipo de investigación de salud suelen generar impacto económico, mediante un retorno de la inversión financiera realizada por los sistemas de salud ${ }^{(15)}$, además de tener mayor probabilidad de citación ${ }^{(16)}$.

En revistas biomédicas en idioma español, el porcentaje de publicaciones sin citaciones es variable. En el periodo 2010-2017, la RPMESP tiene un $40 \%$ de publicaciones originales que no han recibido citaciones, la revista española Gaceta Sanitaria entre 2007 y 2010 tuvo un $48,0 \%{ }^{(14)}$ de publicaciones originales no citadas, similar a la proporción de la RPSP $(48,9 \%)$. El principal factor asociado a la citación de una publicación es el factor de impacto de la revista ${ }^{(17-19)}$, las publicaciones en inglés también tienen un mayor número de citaciones que aquellas publicadas en otros idiomas ${ }^{(20)}$. También se ha identificado que el número de años desde la publicación de un artículo está asociado con un mayor número de citaciones, publicaciones del 2007 tuvieron cinco veces mayor probabilidad de ser citados comparados con aquellos publicados el $2010^{(14)}$.

En ocho años de publicación, el número de citas recibidas por publicaciones originales de la RPMESP fue de 945, mayor al número de citas obtenido entre 2002 y 2009, periodo donde 182 documentos citados generaron un total de 396 citas. ${ }^{(6)}$
Entre 2010 y 2017, la RPSP alcanzó 3485 citas, mientras que la revista de Saude Publica publicada por la Universidad de Sao Paulo llegó a 5808 citaciones. Si bien no es posible comparar este número de citas con revistas de mayor factor de impacto y del cuartil uno en el campo de la salud pública a nivel mundial; mostramos de manera referencial el número de citas que entre 1990 y 2014 recibieron las revistas más influyentes en salud pública como American Journal of Public Health o Social Science \& Medicina, con 302196 y 281663 citas, respectivamente ${ }^{(21)}$. Además, el periodo de análisis de las cifras mostradas corresponde a un mayor periodo de estudio (24 años), y fueron obtenidas de otra base de datos (Web of Science); sin embargo, la principal explicación de la diferencia en el número de citas sería el factor de impacto de dichas revistas y su prestigio a nivel mundial.

La métrica de impacto de la RPMESP según SJR tuvo un incremento en 0,1 entre el 2010 y el 2017, comparando dicho métrica con los de la RPSP encontramos diferencias marcadas (RPMESP versus RPSP; 0,13 y 0,37 en 2010, 0,19 y 0,44 en 2011 y 0,17 y 0,39 en 2012). EI SJR fue menor que los otros indicadores de impacto (CiteScore y SciELO) en todos los años analizados. A diferencia del CiteScore que también es calculado considerando un periodo de tres años, el SJR brinda mayor énfasis a citaciones realizadas por publicaciones en revistas de alto factor de impacto ${ }^{(22)}$. Es probable que las citaciones recibidas por las publicaciones de la RPMESP hayan sido realizadas en revistas de prestigio o importancia moderada o baja, o por autores que publican investigación en idioma español (14). 
Podría considerarse como limitación del estudio, el sólo incluir artículos originales y originales breve como indicadores de investigación original, cuando la RPMESP permite las cartas al editor de tipo científicas, las cuales pueden presentar resultados preliminares de investigación, y son susceptibles de ser citados. Otra limitación, es que el análisis de citas se obtuvo a partir de la base de datos Scopus, lo cual refleja sólo la citación recibida por otros artículos publicados en revistas indizadas en dicha base de datos, lo cual puede no reflejar el verdadero número de citaciones de las publicaciones de la RPMESP.

En conclusión, en el periodo 2010-2017, la RPMESP publicó 1045 documentos, de los cuales el $26,1 \%$ y $14,0 \%$ fueron artículos originales y originales breves. Las instituciones peruanas corresponsales de mayor contribución fueron la UPCH, UNMSM y el INS. La filiación país del autor corresponsal con mayor participación fue Perú. Nueve de cada diez investigaciones originales publicadas usaron diseños observacionales, los cuales principalmente estuvieron centrados en medir la magnitud y distribución de alguna enfermedad, y explorar determinantes de dichos problemas de salud. Las métricas de impacto obtenidos de CiteScore y SciELO muestran una tendencia ascendente especialmente desde 2014, mientras que para el SJR se evidencia un patrón estacionario.

Recomendamos que la RPMSP implemente estrategias que permita mejorar sus indicadores bibliométricos, entre ellos incrementar la captación de estudios experimentales o que empleen diseños diferentes al observacional, así como estudios de investigación traslacional, de desarrollo tecnológico, o de evaluación del impacto de intervenciones en salud, entre otros. Otro aspecto, es reforzar los criterios de selección de las propuestas de manuscritos en base a su potencial de citación futura, lo cual está vinculado con la relevancia de la publicación, así como con la validez científica de los resultados reportados. Otra estrategia a corto plazo para incrementar el número de citaciones podría ser la publicación en idioma inglés, así como brindar la posibilidad de recibir manuscritos en dicho idioma. Por último, si bien las publicaciones y citaciones reflejan los niveles de producción científica, consideramos importante proponer otros indicadores que permitan visibilizar la contribución de las publicaciones de la RPMESP en las políticas sanitarias; también sugerimos evaluar la dinámica de colaboración entre filiaciones institucionales y país de lo publicado en la RPMESP, así como realizar un análisis temático de sus publicaciones.

Contribuciones de los autores: FRR y CCS han participado en la concepción, redacción del manuscrito y aprobación de la versión final. FRR realizó la recolección de los datos, análisis de datos y redacción del primer borrador del manuscrito.

Fuentes de financiamiento: Instituto Nacional de Salud

Conflictos de interés: Los autores forman parte del Comité Editorial de la Revista Peruana de Medicina Experimental y Salud Pública, y pertenecen al Instituto Nacional de Salud.

\section{REFERENCIAS BIBLIOGRÁFICAS}

1. Bornmann L, Leydesdorff L. Scientometrics in a changing research landscape: Bibliometrics has become an integral part of research quality evaluation and has been changing the practice of research. EMBO Rep. 2014;15(12):1228-32. doi: 10.15252/ embr.201439608.

2. Sanz-Valero J, Casterá VT, Wanden-Berghe C. Estudio bibliométrico de la producción científica publicada por la Revista Panamericana de Salud Pública/ Pan American Journal of Public Health en el período de 1997 a 2012. Rev Panam Salud Publica. 2014;25(2):81-8.

3. Krauskopf E. A bibiliometric analysis of the Journal of Infection and Public Health: 2008-2016. J Infect Public Health. 2018;11(2):224-229. doi: 10.1016/j.jiph.2017.12.011.

4. Pereira JC. [Revista de Saúde Pública: forty years of Brazilian scientific production]. Rev Saude Publica. 2006
Aug;40 Spec no.:148-59. [Article in Portuguese]

5. Cooper ID. Bibliometrics basics. J Med Libr Assoc. 2015;103(4):217-8. doi: 10.3163/1536-5050.103.4.013.

6. Huamaní C. Análisis de la producción, visibilidad y citación de la Revista Peruana de Medicina Experimental y Salud Pública, 2002 - 2009. Rev Peru Med Exp Salud Pública. 2010;27(3):367-72. doi: http://dx.doi. org/10.17843/rpmesp.2010.273.1494.

7. World Health Organization. The WHO strategy on research for health [Internet]. Geneva: WHO; 2012 [citado el 25 de abril de 2018]. Disponible en: http://www.who.int/ phi/WHO_Strategy_on_research_ for_health.pdf

8. Papatheodorou SI, Trikalinos TA, Ioannidis JPA. Inflated numbers of authors over time have not been just due to increasing research complexity. J Clin Epidemiol. 2008;61(6):546-51. doi: 10.1016/j.jclinepi.2007.07.017.
9. Železnik D, Blažun Vošner H, Kokol P. A bibliometric analysis of the Journal of Advanced Nursing, 1976-2015. J Adv Nurs. 2017;73(10):2407-19. doi: 10.1111/jan.13296.

10. Huamaní C, Mayta-Tristán P. Producción científica peruana en medicina y redes de colaboración, análisis del Science Citation Index 2000-2009. Rev Peru Med Exp Salud Pública. 2010;27(3):315-25. doi: http://dx.doi. org/10.17843/rpmesp.2010.273.1488.

11. Consejo Nacional de Ciencia, Tecnología e Innovación Tecnológica CONCYTEC. Principales indicadores bibliométricos de la actividad científica peruana, 2006-2011. Lima, Perú: CONCYTEC; 2014

12. Bonfill X, Osorio D, Posso M, Solà I, Rada G, Torres A, et al. Identification of biomedical journals in Spain and Latin America. Health Inf Libr J. 2015;32(4):276-86. doi: 10.1111/ hir.12110. 
13. Romani F, Roque-Henríquez J, Vasquez Loarte T, Mormontoy Calvo H, Vásquez Soplopuco H. Análisis bibliométrico de la producción científica sobre las agendas nacionales de investigación en el Perú 2011-2014. An Fac med. 2016;77(3):241-9.

14. Ruano-Ravina A, Álvarez-Dardet C. Evidence-based editing: factors influencing the number of citations in a national journal. Ann Epidemiol. 2012;22(9):649-53. doi: 10.1016/j.annepidem.2012.06.104.

15. Masters R, Anwar E, Collins B, Cookson R, Capewell S. Return on investment of public health interventions: a systematic review. J Epidemiol Community Health. 2017;71(8):827-34. doi: 10.1136/jech-2016-208141.

16. Wolfenden L, Milat AJ, Lecathelinais C, Skelton E, Clinton-McHarg T, Williams $\mathrm{C}$, et al. A bibliographic review of public health dissemination and imple- mentation research output and citation rates. Prev Med Rep. 2016;4:441-3. doi: 10.1016/j.pmedr.2016.08.006.

17. Filion KB, Pless IB. Factors related to the frequency of citation of epidemiologic publications. Epidemiol Perspect Innov. 2008;5(1):3. doi: 10.1186/1742-5573-5-3.

18. Weale AR, Bailey M, Lear PA. The level of non-citation of articles within a journal as a measure of quality: a comparison to the impact factor. BMC Med Res Methodol. 2004;4(1):14.

19. Yaminfirooz M, Ardali F. Identifying the Factors Affecting Papers' Citability in the Field of Medicine: an Evidencebased Approach Using 200 Highly and Lowly-cited Papers. Acta Inform Med. 2018;26(1):10-4. doi: 10.5455/ aim.2018.26.10-14.

20. Di Bitetti MS, Ferreras JA. Publish (in English) or perish: The effect on citation rate of using languages other than
English in scientific publications. Ambio. 2017;46(1):121-7. doi: 10.1007/ s13280-016-0820-7.

21. Merigó JM, Núñez A. Influential journals in health research: a bibliometric study. Glob Health. 2016;12(1):46. doi: 10.1186/s12992-016-0186-4.

22. Agarwal A, Durairajanayagam D, Tatagari S, Esteves S, Harlev A, Henkel R, et al. Bibliometrics: tracking research impact by selecting the appropriate metrics. Asian J Androl. 2016;18(2):296-309. doi: 10.4103/1008-682X.171582.

Correspondencia: Franco Romani Romani Dirección: Calle Capac Yupanqui 1400, Jesús María, Lima-Perú.

Teléfono: 990630678

Correo electrónico:fromani@ins.gob.pe

\title{
Ahora nuestra revista incluye:
}

\author{
$\checkmark$ Publicación anticipada \\ $\checkmark$ Compartiendo publicaciones científicas con el ciudadano \\ $\checkmark$ Videos de presentaciones conjuntas del Instituto Nacional de \\ Salud y la Academia Nacional de Medicina \\ $\checkmark$ Galería fotográfica y videos
}

位htps://rpmesp.ins.gob.pe

MINISTERIO DE SALUD

Instituto Nacional de Salud

Síguenos en:

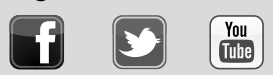

